\title{
Micro-particle swarm optimizer for solving high dimensional optimization problems
}

\section{( $\mu$ PSO for high dimensional optimization problems)}

By

\author{
Tony Huang $^{1}$ and Ananda Sanagavarapu Mohan ${ }^{1 *}$
}

${ }^{1}$ Microwave and Wireless Technology Research Laboratory Information and Communication Group, Faculty of Engineering, University of Technology, Sydney

PO Box 123, Broadway, NSW 2007, Australia

\footnotetext{
*Corresponding author. Address:

Microwave and Wireless Technology Research Laboratory, Information and Communication Group, Faculty of Engineering, University of Technology, Sydney PO Box 123, Broadway, NSW 2007, Australia.

E-mail Address: $\quad$ ananda@eng.uts.edu.au
} 
Abstract —A simple and powerful micro-particle swarm optimizer ( $\mu \mathrm{PSO})$ is presented. For high dimensional optimization problems, the proposed $\mu$ PSO needs only a small population to outperform the standard particle swarm optimizer (PSO) that uses a larger population. This superior performance is confirmed by simulation results for different high dimensional test functions.

Key words - Evolutionary computation; High dimensional optimization problems; Microparticle swarm optimizer; Optimization methods; Particle swarm optimizer. 


\section{Introduction}

The process of optimization has assumed greater importance in many real life engineering problems. Recently, the particle swarm optimizer (PSO) proposed by Eberhart and Kennedy [1] has gained a huge popularity due to its algorithmic simplicity and effectiveness. Although it has been reported that PSO is not sensitive to the population size [2], this, however, is not true in terms of computational cost for evaluating the fitness of the entire population of particles. Parametric studies have shown that a population size of 30 particles is suitable for solving most of the low dimensional problems [3]. For high dimensional problems (e.g. $N \geq 100$ ), on the other hand, the PSO performance starts to suffer due to the curse of dimensionality [4]. To minimize the impact of this problem while maintaining a low computational cost, this paper presents a novel micro-particle swarm optimizer ( $\mu \mathrm{PSO}$ ) for solving high dimensional optimization problems.

The paper is organized as follows. In Section 2, the concept of $\mu$ PSO and its operations are described. Section 3 presents simulation results on the use of $\mu$ PSO for solving high dimensional optimization problems. Comparisons on the optimization performance between $\mu \mathrm{PSO}$ and standard PSO are also provided to highlight the effectiveness of the proposed $\mu \mathrm{PSO}$ technique. Finally, the conclusion of this paper is given in Section 4.

\section{Micro-Particle swarm optimizer}

The concept of $\mu \mathrm{PSO}$ is analogous to the idea behind the micro-genetic algorithm ( $\mu \mathrm{GA})$, where the optimization is done by a small population size of 3 to 5 particles, and 
a set of restart operations are executed after the population has converged. The convergence of population is defined as the percentage of particles that achieve a particular value of standard deviation on their locations which is less than a predefined convergence threshold. If the population converges to a solution that is inferior to the best available solution, the inferior solution is blacklisted for future searches and the particles are prevented from converging to the same inferior solution again.

To achieve this prevention and taking the consideration that the standard PSO does not have guaranteed convergence properties [5], the velocity update equation used in $\mu$ PSO is a modified version of the guaranteed convergence PSO (GCPSO) [5], that is:

$$
\begin{aligned}
& v_{j, n}(t+1)=w v_{j, n}(t)+c_{1} r_{1}\left[p_{j, b e s t}-x_{j, n}(t)\right]+c_{2} r_{2}\left[g_{\text {best }}-x_{j, n}(t)\right]+r e p_{j, n}(t) \\
& v_{g, n}(t+1)=w v_{g, n}(t)-x_{g, n}(t)+g_{\text {best }}+\rho(t)\left(1-2 r_{3}\right)+r e p_{g, n}(t)
\end{aligned}
$$

where $v_{g, n}$ and $v_{j, n}$ refers to the velocities within the $n^{\text {th }}$ dimension of the global best and the $j^{\text {th }}$ remaining particle, respectively. $w$ is particles' inertia weight which is a constant that would either return to its initial value or increase by a small percentage $\beta$ (e.g. $\beta=$ $10 \%$ ) whenever the population is converged to a better or inferior solution, respectively. $c_{1}$ and $c_{2}$ are the accelerating constants, and $r_{1}, r_{2}$ and $r_{3}$ are uniformly distributed random variables in the range of $[0,1] . \rho(t)$ is a scaling factor as defined in [5], and $\operatorname{rep}_{i, n}(t)$ is the total repulsion experienced within the $n^{\text {th }}$ dimension of the $i^{\text {th }}$ particle from the blacklisted solutions.

In (1) and (2), the repulsion is used to repel particles away from blacklisted solutions. Unlike common approaches where particles are repelled immediately when they are inside a predefined space surrounding blacklisted solutions, the repulsion in $\mu \mathrm{PSO}$ is computed based on the lines of well known Coulomb's law. We have replaced 
the electrostatic constant with the dynamic range of the problem space, $D$, and both the particles and blacklisted solutions are assumed to be charges of same polarity with unity magnitude. Hence, for the $i^{\text {th }}$ particle, the repulsion experienced from $L$ blacklisted solutions can be described as:

$$
\boldsymbol{r e p}_{i}=D \sum_{l=1}^{L} \frac{\boldsymbol{d}_{l i}}{\left\|\boldsymbol{d}_{l i}\right\|^{m+1}}
$$

where $\boldsymbol{d}_{l i}=\boldsymbol{x}_{\boldsymbol{i}}-\hat{\boldsymbol{x}}_{\boldsymbol{l}}$ is a vector pointing from the blacklisted solution $l$ to the $i^{\text {th }}$ particle. $\boldsymbol{x}_{\boldsymbol{i}}$ and $\hat{\boldsymbol{x}}_{l}$ are the position vectors of the $i^{\text {th }}$ particle and the blacklisted solution $l$, respectively. As can be seen from (3), the repulsion is inversely proportional to $\left\|\boldsymbol{d}_{l i}\right\|^{m+1}$, thus the amount of repulsion experienced by the particles at a particular position can be controlled by the parameter $m$. Our preliminary studies have indicated that $m=20$ is an appropriate value.

With this modified Coulomb's law approach, two immediate advantages can gained over other commonly used approaches [6]. The first is the removal of the burden for determining the suitable size of space needed to enclose the blacklisted solutions and the amount of repulsion needed to repel the particles, as these parameters are extremely difficult to determine for high dimensional problems. Another advantage is the flexibility of controlling the repulsion on particles through the use of parameter $m$. By properly choosing the value of $m$, we can prevent particles from converging to the blacklisted solutions while allowing them to explore the nearby surrounding space without experiencing too much unnecessary repulsion. At this point it should be emphasized that although the analogy of identical charges has been used for particles and blacklisted solutions, but the repulsion should only exist between particles and blacklisted solutions 


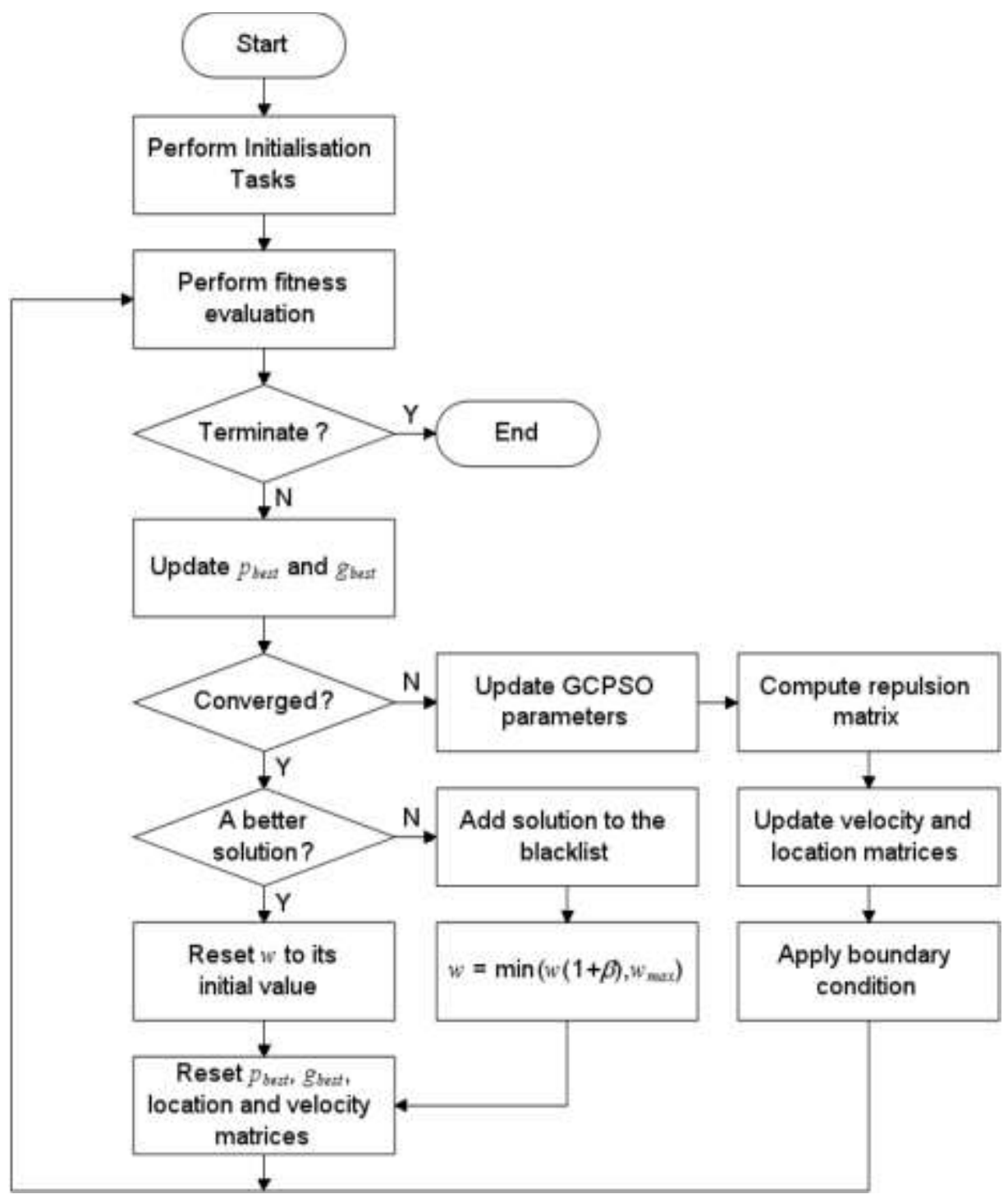

Fig. 1. Flowchart of the proposed $\mu \mathrm{PSO}$ operations.

and not among particles themselves. Fig. 1 shows the flow of the overall operation of the proposed $\mu \mathrm{PSO}$.

\section{Simulation results}

To demonstrate the effectiveness of $\mu \mathrm{PSO}$, five well known test functions have been chosen to evaluate the performance between $\mu \mathrm{PSO}$ and the standard PSO. The five test functions are: 
Rastigrin function

$$
f_{1}(x)=\sum_{n=1}^{N}\left[x_{n}^{2}-10 \cos \left(2 \pi x_{n}\right)+10\right],-2 \leq x_{n} \leq 2
$$

Rosenbrock function

$$
f_{2}(x)=\sum_{n=1}^{N-1}\left[100\left(x_{n+1}-x_{n}^{2}\right)^{2}+\left(x_{n}-1\right)^{2}\right],-2 \leq x_{n} \leq 2
$$

Griewank function

$$
f_{3}(x)=1+\frac{1}{4000} \sum_{n=1}^{N} x_{n}^{2}-\prod_{n=1}^{N} \cos \left(\frac{x_{n}}{\sqrt{n}}\right),-10 \leq x_{n} \leq 10
$$

Schwefel function

$$
f_{4}(x)=418.9829 n+\sum_{n=1}^{N} x_{n} \sin \left(\sqrt{\left|x_{n}\right|}\right),-500 \leq x_{n} \leq 500
$$

Ackley function

$$
f_{5}(x)=20+e-20 \exp \left(-0.2 \sqrt{\frac{1}{N} \sum_{n=1}^{N} x_{n}^{2}}\right)-\exp \left(\frac{1}{N} \sum_{n=1}^{N} \cos \left(2 \pi x_{n}\right)\right),-2 \leq x_{n} \leq 2
$$

The dimension of all five test functions have been set to 500, i.e. $N=500$. The $\mu \mathrm{PSO}$ is implemented with the parameters shown in Table 1. The standard PSO is implemented using the description given in [3], and for the purpose of comparison we have set the PSO population size to 3 and 30 particles. Both $\mu$ PSO and PSO are set to terminate after 3000 fitness evaluations, and the final result is taken as the average of 1000 independent simulation runs. 
Table 1

Parameters used to implement the $\mu \mathrm{PSO}$ for our investigations.

\begin{tabular}{ll}
\hline Parameters & Parameter Values \\
\hline Population Size & 3 \\
Initial $w$ & 0.2 \\
$w_{\max }$ & 0.9 \\
$c_{1}$ & 1.49 \\
$c_{2}$ & 1.49 \\
Population Convergence & $80 \%$ \\
Convergence Threshold & 0.001 \\
$m$ & 20 \\
$\beta$ & $10 \%$ \\
\hline
\end{tabular}

Table 2

Optimization results after 1000 independent simulation runs.

\begin{tabular}{|c|c|c|c|c|c|c|}
\hline \multirow{2}{*}{$\begin{array}{c}\text { Test } \\
\text { functions } \\
(N=500)\end{array}$} & \multicolumn{2}{|c|}{$\begin{array}{c}\mu \mathrm{PSO} \\
3 \text { particles } \\
\end{array}$} & \multicolumn{2}{|c|}{$\begin{array}{c}\text { PSO } \\
3 \text { particles } \\
\end{array}$} & \multicolumn{2}{|c|}{$\begin{array}{c}\text { PSO } \\
30 \text { particles }\end{array}$} \\
\hline & Avg. & Std. Dev. & Avg. & Std. Dev. & Avg. & Std. Dev. \\
\hline$f_{1}(x)$ & $1,603.81$ & 301.44 & $3,897.29$ & 256.93 & $3,667.24$ & 424.92 \\
\hline$f_{2}(x)$ & $14,165.89$ & $4,567.85$ & $119,367.20$ & $9,883.42$ & $44,358.45$ & $3,641.42$ \\
\hline$f_{3}(x)$ & 1.40 & 0.11 & 3.57 & 0.13 & 2.38 & 0.07 \\
\hline$f_{4}(x)$ & $123,536.23$ & $5,529.60$ & $174,982.59$ & $3,472.15$ & $160,014.07$ & $6,151.62$ \\
\hline$f_{5}(x)$ & 4.13 & 0.14 & 4.94 & 0.08 & 4.17 & 0.07 \\
\hline
\end{tabular}

Figs. 2 to 6 show the comparison of optimization performances for the five test functions, and Table 2 shows the summary of our simulation results. It can be seen that $\mu \mathrm{PSO}$ provides a much superior optimization performance than the standard PSO. 


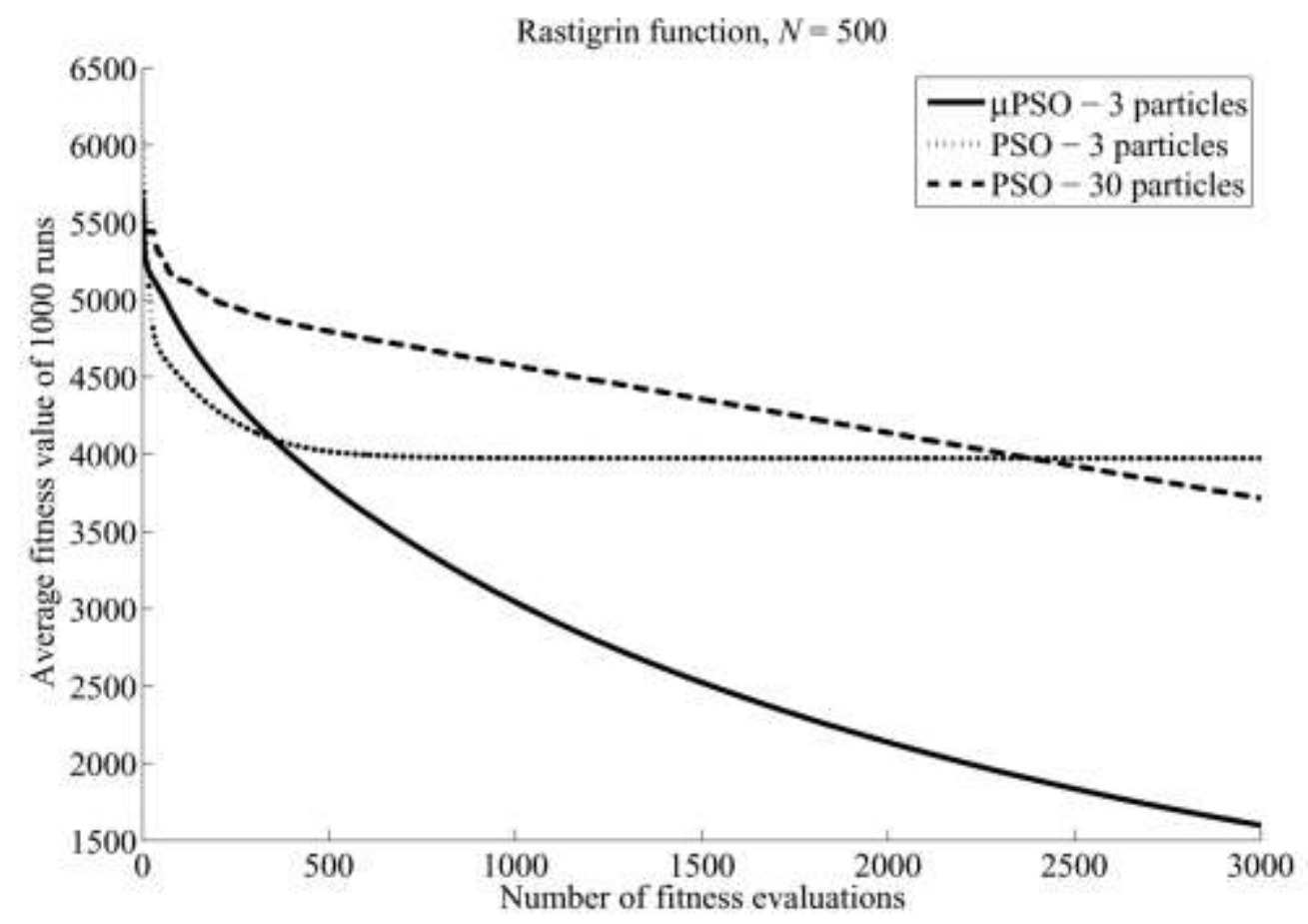

Fig. 2. Comparison of the optimization performance between $\mu$ PSO and PSO for the Rastigrin function of dimension 500 .

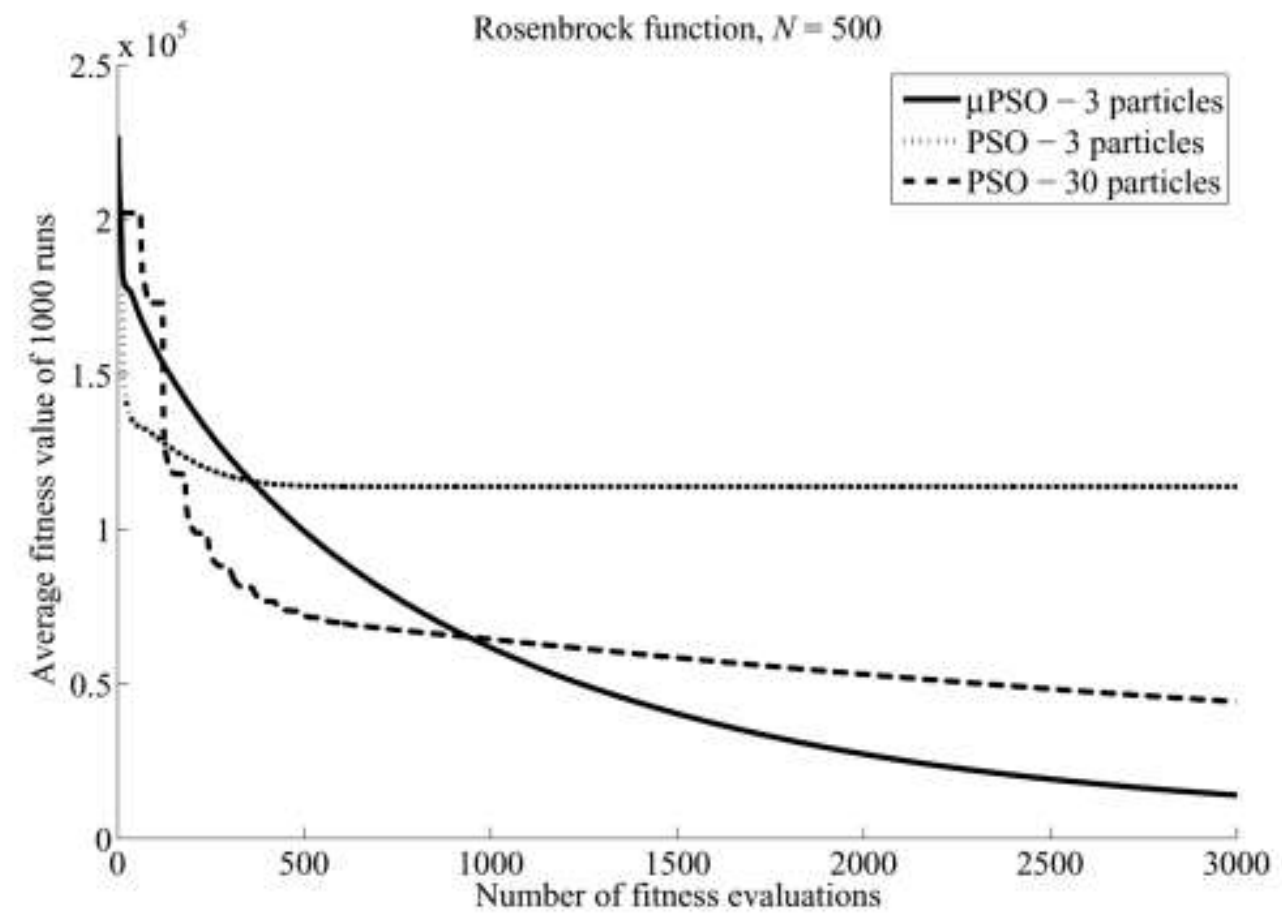

Fig. 3. Comparison of the optimization performance between $\mu$ PSO and PSO for the Rosenbrock function of dimension 500. 


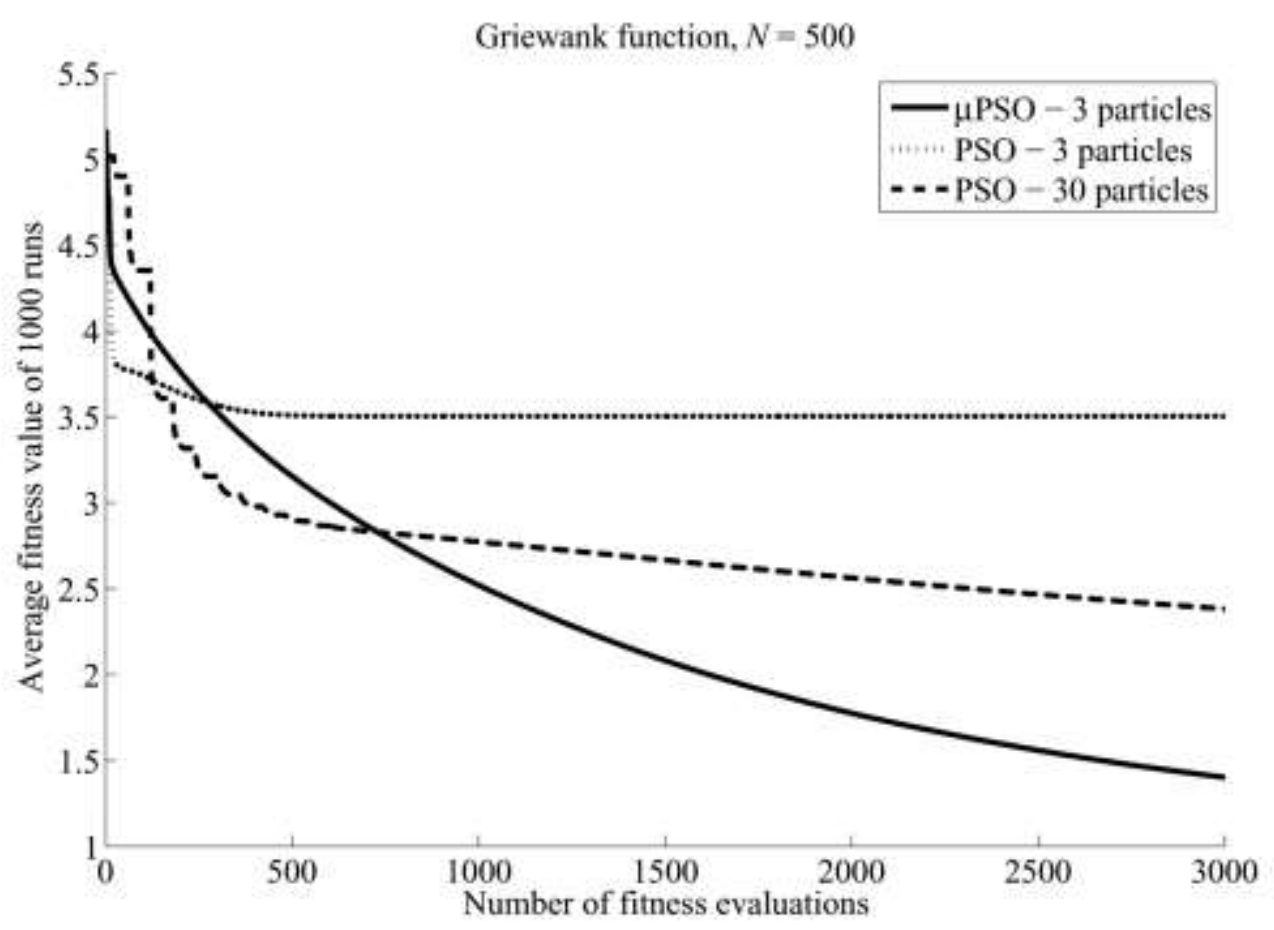

Fig. 4. Comparison of the optimization performance between $\mu$ PSO and PSO for the Griewank function of dimension 500.

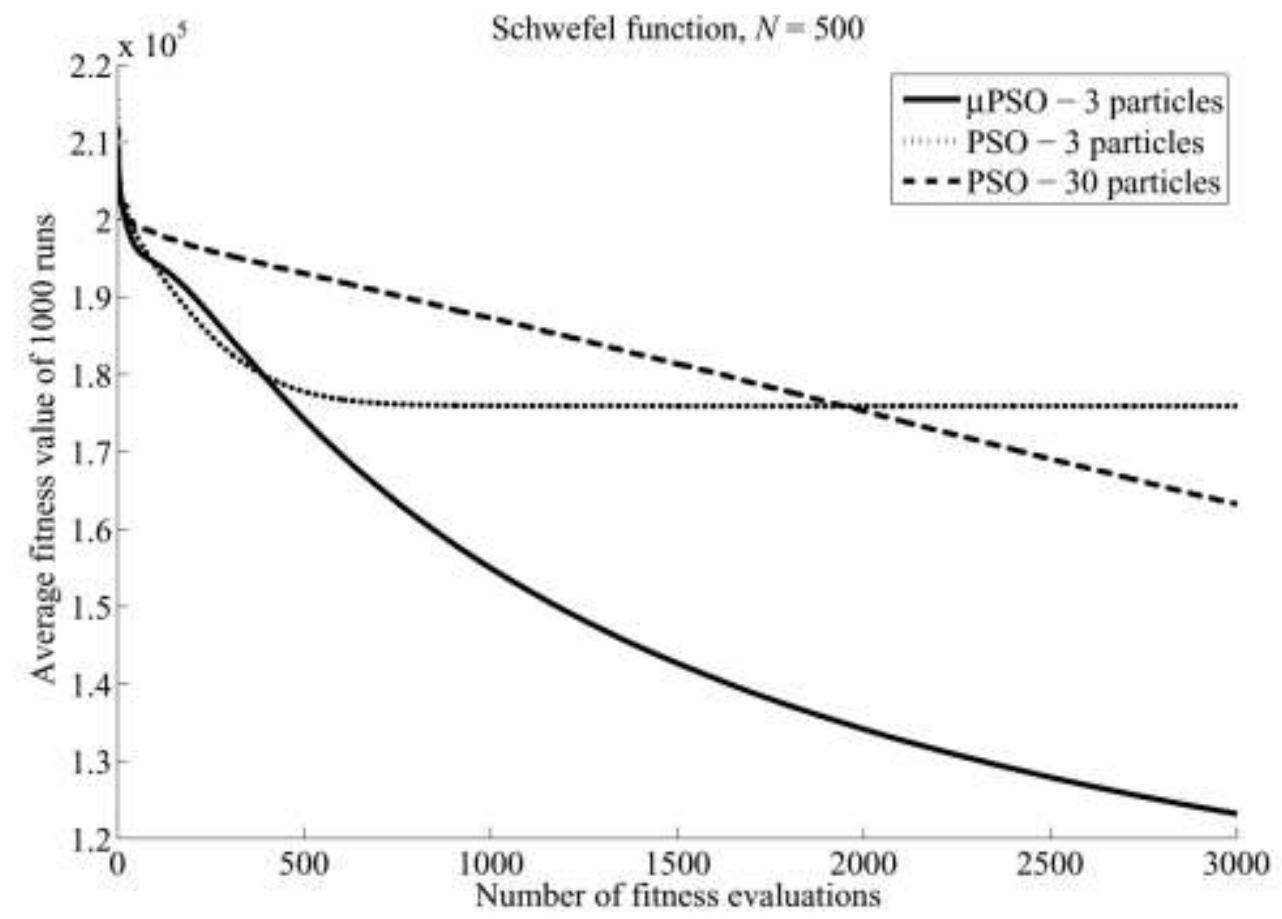

Fig. 5. Comparison of the optimization performance between $\mu$ PSO and PSO for the Schwefel function of dimension 500 . 


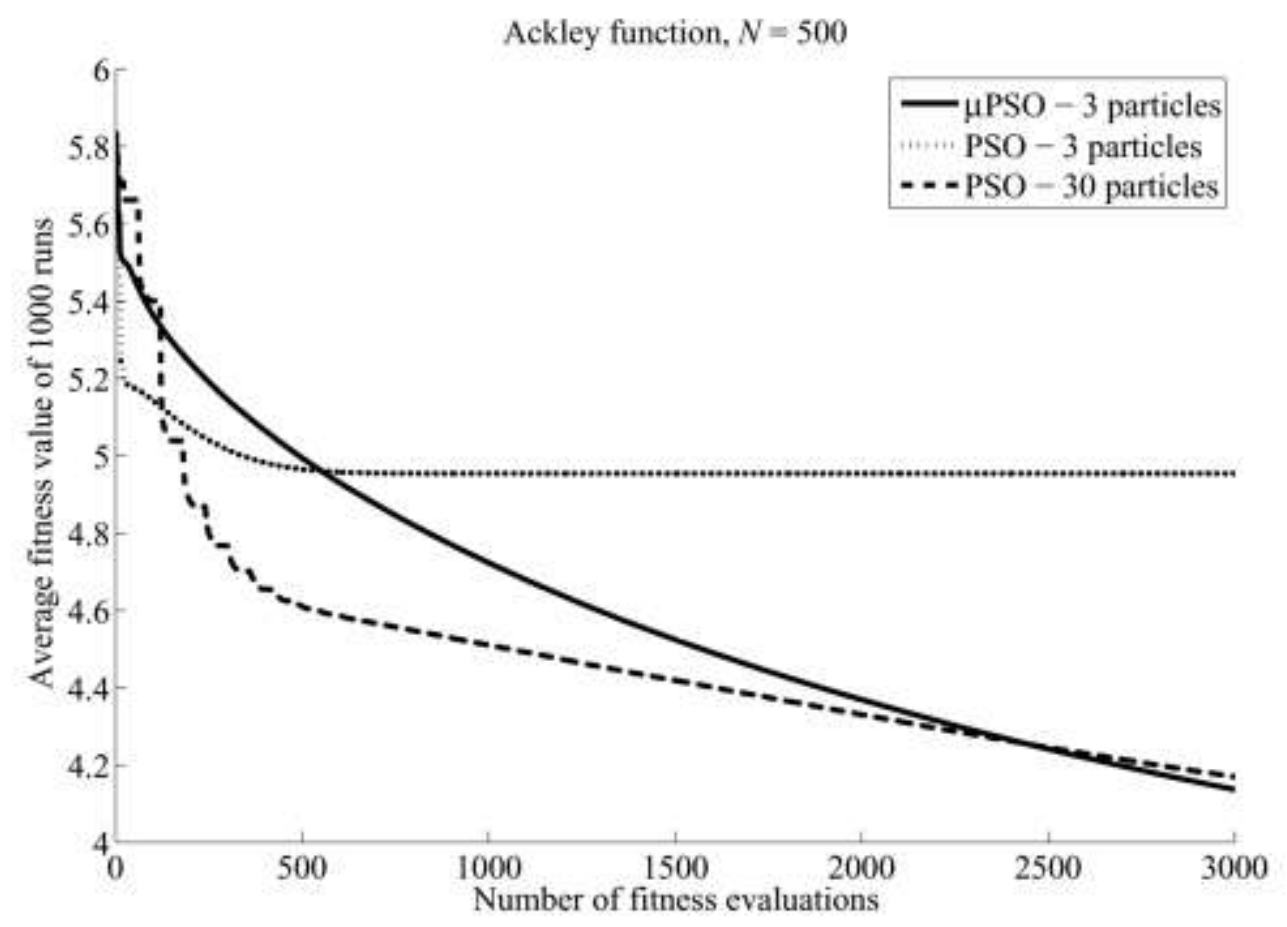

Fig. 6. Comparison of the optimization performance between $\mu$ PSO and PSO for the Ackley function of dimension 500 .

\section{Conclusion}

In this paper, we have for the first time proposed a new algorithm called the micro-particle swarm optimizer ( $\mu$ PSO) for solving high dimensional optimization problems. The performance of the $\mu \mathrm{PSO}$ has also been conformed against various well known test functions. For high dimensional optimization problems, the standard PSO often requires a large number of particles in order to find the global minimum. This large population size would, however, increases the computational cost in evaluating the fitness of all potential solutions that are represented by the particles. The advantage of the proposed $\mu \mathrm{PSO}$ arises from having a small population size, which reduces drastically the computational cost associated with fitness evaluation. In addition, even with its small population size, our simulation results have shown that the proposed $\mu \mathrm{PSO}$ is more 
suitable technique for solving high dimensional optimization problems than the standard PSO of a larger population size.

\section{Acknowledgement}

The work reported in this paper is funded by the Australian Research Council through a Discovery Project Grant DP0346540.

\section{References}

[1] R. C. Eberhart and J. Kennedy, Particle swarm optimization, in Proc. IEEE Conf. Neural Networks IV, Piscataway, NJ, 1995, pp. 1942-1948.

[2] Y. Shi and R. C. Eberhart, Empirical study of particle swarm optimization, in Proc. Cong. on Evolutionary Computing, Washington, DC, 1999, pp. 1945-1950.

[3] A. Carlisle and G. Dozier, An off-the-shelf pso, in Proc. Workshop Particle Swarm Optimization, Indianapolis, IN, 2001.

[4] R. E. Bellman, Adaptive control processes, Princeton University Press, Princeton, NJ, 1961.

[5] E. S. Peer, F. van den Bergh, and A. P. Engelbrecht, Using neighborhoods with the guaranteed convergence PSO, in Proc. IEEE Swarm Intelligence Symp., Indianapolis, IN, 2003, pp. 235-242.

[6] K. E. Parsopoulos and M. N. Vrahatis, On the computation of all global minimizes through particle swarm optimization, IEEE Trans. Evol. Comput. 8 (3):211-224 (2004). 
Table 1

Parameters used to implement the $\mu \mathrm{PSO}$ for our investigations.

\begin{tabular}{ll}
\hline Parameters & Parameter Values \\
\hline Population Size & 3 \\
Initial $w$ & 0.2 \\
$w_{\max }$ & 0.9 \\
$c_{1}$ & 1.49 \\
$c_{2}$ & 1.49 \\
Population Convergence & $80 \%$ \\
Convergence Threshold & 0.001 \\
$m$ & 20 \\
$\beta$ & $10 \%$ \\
\hline
\end{tabular}


Table 2

Optimization results after 1000 independent simulation runs.

\begin{tabular}{|c|c|c|c|c|c|c|}
\hline \multirow{2}{*}{$\begin{array}{c}\text { Test } \\
\text { functions } \\
(N=500) \\
\end{array}$} & \multicolumn{2}{|c|}{$\begin{array}{c}\mu \mathrm{PSO} \\
3 \text { particles } \\
\end{array}$} & \multicolumn{2}{|c|}{$\begin{array}{c}\text { PSO } \\
3 \text { particles } \\
\end{array}$} & \multicolumn{2}{|c|}{$\begin{array}{c}\text { PSO } \\
30 \text { particles } \\
\end{array}$} \\
\hline & Avg. & Std. Dev. & Avg. & Std. Dev. & Avg. & Std. Dev. \\
\hline$f_{1}(x)$ & $1,603.81$ & 301.44 & $3,897.29$ & 256.93 & $3,667.24$ & 424.92 \\
\hline$f_{2}(x)$ & $14,165.89$ & $4,567.85$ & $119,367.20$ & $9,883.42$ & $44,358.45$ & $3,641.42$ \\
\hline$f_{3}(x)$ & 1.40 & 0.11 & 3.57 & 0.13 & 2.38 & 0.07 \\
\hline$f_{4}(x)$ & $123,536.23$ & $5,529.60$ & $174,982.59$ & $3,472.15$ & $160,014.07$ & $6,151.62$ \\
\hline$f_{5}(x)$ & 4.13 & 0.14 & 4.94 & 0.08 & 4.17 & 0.07 \\
\hline
\end{tabular}




\section{Figure Captions:}

Fig. 1. Flowchart of the proposed $\mu$ PSO operations.

Fig. 2. Comparison of the optimization performance between $\mu$ PSO and PSO for the Rastigrin function of dimension 500.

Fig. 3. Comparison of the optimization performance between $\mu$ PSO and PSO for the Rosenbrock function of dimension 500 .

Fig. 4. Comparison of the optimization performance between $\mu$ PSO and PSO for the Griewank function of dimension 500 .

Fig. 5. Comparison of the optimization performance between $\mu$ PSO and PSO for the Schwefel function of dimension 500 .

Fig. 6. Comparison of the optimization performance between $\mu$ PSO and PSO for the Ackley function of dimension 500 . 